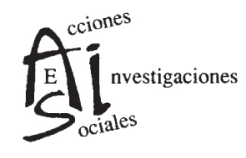

\title{
Aproximación sociolaboral a la discapacidad. ¿Alternativas para el emprendimiento?
}

\author{
Ma Belén García-Palma \\ Universidad de Murcia, Dpto. Sociología \\ mbelengp@um.es \\ Salvador Manzanera-Román \\ Universidad de Murcia, Dpto. SocIología \\ smanzanera@um.es \\ Pilar Ortiz García \\ Universidad de Murcia, Dpto. Sociología \\ portizg@um.es \\ Ángel J. Olaz Capitán \\ Universidad de Murcia, Dpto. Sociología \\ olazcapi@um.es
}

Resumen: Este trabajo tiene como finalidad mostrar algunos rasgos distintivos sobre la situación sociolaboral de las personas con discapacidad y realizar una aproximación al emprendimiento como alternativa profesional, 
valorando el papel de los poderes públicos en su promoción y desarrollo. Este estudio se enmarca dentro del proyecto Discapacidad y emprendimiento. Análisis competencial (CSO2016-75818-R), financiado por el Ministerio de Economía y Competitividad, cuyo objetivo principal consiste en analizar el papel que desempeñan las competencias -entendidas como un conjunto de conocimientos, capacidades y habilidades- en personas que presentan discapacidad física y/o sensorial, en el diseño, desarrollo y actuación en el ámbito del emprendimiento. En definitiva, el proyecto permite profundizar en el conocimiento de cuáles son los aspectos con mayor influencia sobre el emprendimiento de las personas con discapacidad y, también, qué posibles opciones podría abordarse en el legítimo intento de visibilizar a este colectivo, sin el cual no sería posible entender nuestro actual modelo de sociedad.

Palabras clave: Desempleo, barreras, mercado de trabajo, políticas de empleo.

\section{A socio-labour approach to disability. New options for entrepreneurship?}

Abstract: The aim of this paper is to show some distinctive features about the social and labour situation of people with disabilities and to make an approach to entrepreneurship as a professional alternative, valuing the role of public authorities in their promotion and development. This study is part of the Disability and entrepreneurship project. Competencial analysis (CSO2016-75818-R), funded by the Economy and Finance Ministry, whose main goal is to analyze the role played by competences -understood as a set of knowledge, skills and abilities- in people with physical and / or sensory disabilities, in the design, development and performance in the field of entrepreneurship. In short, the project allows to deep in the knowledge of which aspects have the greatest influence on the entrepreneurship of people with disabilities and, also, what possible options could be addressed in the legitimate attempt to make this group visible, without which it would not be possible to understand our current model of society.

Key words: Unemployment, barriers, labour market, labour policies. 


\section{Aproximación sociolaboral a la discapacidad. ¿Alternativas para el emprendimiento?}

$M^{a}$ Belén

García-Palma

Salvador

Manzanera-Román

Pilar

Recibido: 12/1/2018

Aceptado: 4/5/2018

Ortiz García

Ángel J.

Olaz Capitán

\section{INTRODUCCIÓN. PLANTEAMIENTO DE LA INVESTIGACIÓN.}

Este trabajo forma parte del proyecto de $I+D+i$, financiado por el Ministerio de Economía y Competitividad, denominado Discapacidad y emprendimiento. Análisis competencial (CSO2016-75818-R) cuyo objetivo principal es conocer el papel que representan las competencias (conocimientos, capacidades y habilidades) de las personas con discapacidad física y/o sensorial a la hora de explicar sus acciones de emprendimiento.

Este estudio pone su foco en las singularidades que el fenómeno del emprendimiento presenta sobre un colectivo notablemente heterogéneo, donde la discapacidad física y sensorial presentan múltiples facetas y manifestaciones, casi tantas, como personas.

Bajo este esquema la hipótesis principal de la investigación se basa en la idea de que las personas con discapacidad se encuentran en una posición netamente desfavorable en comparación con aquellas que no la tienen a la hora de desarrollar una actividad emprendedora. No debe olvidarse que la relevancia del emprendimiento como actividad económica en general es clave como vía de integración social y, por tanto, sugiere la oportunidad de abordar un análisis diferenciado. En este sentido, conocer los elementos moderadores (inhibidores y potenciadores) 
del emprendimiento son clave para el diseño y desarrollo de estrategias orientadas al emprendimiento de este colectivo, "con dificultades de inserción laboral", pero con un notable potencial.

En lo que respecta a este artículo, el objetivo es caracterizar a la población con discapacidad, conocer su posición en el mercado laboral y considerar el papel de las iniciativas públicas en general, y de fomento del emprendimiento en particular, en su integración sociolaboral.

\section{ALGUNAS CUESTIONES PREVIAS.}

El siempre complejo proceso de abordar un concepto como el de discapacidad comporta diferenciar entre un conjunto de términos que, no por estar más o menos relacionados, generan interpretaciones homogéneas. Según la Clasificación Internacional de Deficiencias, Discapacidades y Minusvalías (CIDDM) se distinguen distintos conceptos (OMS, 1997), como son enfermedad, deficiencia, discapacidad o minusvalía. En esta clasificación, la discapacidad se entiende como el modo en que se objetiva la deficiencia en la persona, repercutiendo directamente en su capacidad para la realización de actividades consideradas normales para cualquier sujeto de similares características por razón de edad, género, etc.

Desde otra perspectiva, las personas con discapacidad son aquellas que poseen un grado de ésta superior o igual al $33 \%$, según el procedimiento de valoración establecido por $R D$ 1971/1999 modificado por RD 1364/2012.

De un modo más específico, el Real Decreto 1971/1999, de 23 de diciembre, clasifica la discapacidad en tres grandes grupos: a) Las discapacidades físicas que se encuentran vinculadas con el cuerpo, miembros y órganos; b) Las discapacidades sensoriales asociadas al aparato visual, oído, garganta y todas aquellas estructuras relacionadas con el lenguaje y c) Las discapacidades psíquicas relacionadas con el retraso y/o enfermedad mental.

Según el estudio de la Organización Nacional de Ciegos Españoles, ONCE (2013:28) basada en las encuestas EDAD (Encuesta de Discapacidad, Autonomía y Dependencia) 2008 y EDDES (Encuesta sobre Discapacidades, Deficiencias y Estado de Salud) 
1999, se recogen 44 clases de discapacidad clasificadas en 8 grupos en los que se gradúa la intensidad de la discapacidad.

En cualquier caso, y con independencia de las fuentes, es evidente que la discapacidad condiciona la actividad diaria de la persona, lo que no debería ser un obstáculo insalvable para desarrollar un proceso de emprendimiento ajustado a tales circunstancias.

Una primera aproximación al perfil de la persona con discapacidad, según la encuesta del INE El Empleo de las Personas con Discapacidad - basada en la explotación integrada de la Encuesta de Población Activa y de la Base Estatal de Personas con Discapacidad - revela que en el año 2015 el número de personas en edad de trabajar (de 16 a 64 años) en España era de 30.174.500, de las que 1.774.800 personas poseían el certificado de discapacidad, lo que representa el $5,8 \%$ de la población total en edad laboral. De este total el $57,7 \%$ corresponde a hombres, frente al $42,3 \%$ restante de mujeres.

Por tramos de edad, el colectivo más numeroso se concentra en el intervalo de 45 a 64 años (69,9\%), seguido del tramo de 25 a 44 años $(25,7 \%)$ lo que refleja el impacto de las discapacidades sobrevenidas.

Finalmente, y no por ello menos importante, destaca el menor nivel educativo de las personas con discapacidad con relación a las que no tienen. Por ejemplo, con respecto a los estudios primarios, las primeras registran el $23,9 \%$ frente al $9,6 \%$, de personas con discapacidad y en estudios superiores un $15,1 \%$ frente al 33,2\%, respectivamente.

En otras palabras, se puede caracterizar a la población con discapacidad, por una predominante presencia masculina, de edad media y avanzada y con un nivel de estudios bajo (Tabla 1). 
Tabla 1.

Población en edad de trabajar (de 16 a 64 años) según presencia de discapacidad. 2015.

\begin{tabular}{|c|c|c|c|c|c|c|}
\hline & \multicolumn{2}{|c|}{ Total } & \multicolumn{2}{c|}{$\begin{array}{c}\text { Personas sin } \\
\text { discapacidad }\end{array}$} & \multicolumn{2}{c|}{$\begin{array}{c}\text { Personas con } \\
\text { discapacidad }\end{array}$} \\
\hline Sexo & $\mathbf{N}^{\circ}$ & $\%$ & $\mathbf{N}^{\circ}$ & $\%$ & $\mathbf{N}^{\circ}$ & $\%$ \\
\hline Mujeres & 15.047 .100 & 49,9 & 14.296 .100 & 50,3 & 751.000 & 42,3 \\
\hline Hombres & 15.127 .400 & 50,1 & 14.103 .600 & 49,7 & 1.023 .800 & 57,7 \\
\hline Total & 30.174 .500 & 100 & 28.399 .700 & 100 & 1.774 .800 & 100 \\
\hline Edad & $\mathbf{N}^{\circ}$ & $\%$ & $\mathbf{N}^{\circ}$ & $\%$ & $\mathbf{N}^{\circ}$ & $\%$ \\
\hline $16-24$ & 4.007 .700 & 13,3 & 3.923 .200 & 13,8 & 84.500 & 4,8 \\
\hline $25-44$ & 13.535 .700 & 44,9 & 13.079 .800 & 46,1 & 455.900 & 25,7 \\
\hline $45-64$ & 12.631 .100 & 41,9 & 11.396 .700 & 40,1 & 1.234 .400 & 69,6 \\
\hline Total & 30.174 .500 & 100 & 28.399 .700 & 100 & 1.774 .800 & 100 \\
\hline N. Estudios & $\mathbf{N}^{\circ}$ & $\%$ & $\mathbf{N}^{\circ}$ & $\%$ & $\mathbf{N}^{\circ}$ & $\%$ \\
\hline Analfabetos & 244.100 & 0,8 & 141.700 & 0,5 & 102.400 & 5,8 \\
\hline Primarios & 3.161 .600 & 10,5 & 2.736 .800 & 9,6 & 424.800 & 23,9 \\
\hline Secundaria & 17.076 .400 & 56,6 & 16.096 .400 & 56,7 & 980.000 & 55,2 \\
\hline Superiores & 9.692 .400 & 32,1 & 9.424 .700 & 33,2 & 267.700 & 15,1 \\
\hline Total & 30.174 .500 & 100 & 28.399 .700 & 100 & 1.774 .800 & 100 \\
\hline
\end{tabular}

Fuente: elaboración propia a partir de la operación estadística "El empleo de las personas con discapacidad" (INE).

En relación con el mercado de trabajo, hay un rasgo particular en el colectivo de personas con discapacidad y es su menor actividad. La Tabla 2 muestra que solo un tercio del colectivo es laboralmente activo, 33,9\%, frente al $78,1 \%$ de las personas sin discapacidad, lo que representa un elemento clave a considerar en cualquier medida de promoción de su integración sociolaboral. 
TABLA 2.

Población en edad de trabajar (de 16 a 64 años) en relación con la actividad. 2015.

\begin{tabular}{|c|c|c|c|c|c|c|}
\hline & \multicolumn{2}{|c|}{ Total } & \multicolumn{2}{c|}{$\begin{array}{c}\text { Personas sin } \\
\text { discapacidad }\end{array}$} & \multicolumn{2}{c|}{$\begin{array}{c}\text { Personas con } \\
\text { discapacidad }\end{array}$} \\
\hline & $\mathrm{N}^{\circ}$ & $\%$ & $\mathrm{~N}^{\circ}$ & $\%$ & $\mathrm{~N}^{\circ}$ & $\%$ \\
\hline Activos & 22.767 .100 & 75,5 & 22.166 .000 & 78,1 & 601.100 & 33,9 \\
\hline Inactivos & 7.407 .300 & 24,5 & 6.233 .700 & 21,9 & 1.173 .600 & 66,1 \\
\hline Total & $\mathbf{3 0 . 1 7 4 . 5 0 0}$ & $\mathbf{1 0 0}$ & $\mathbf{2 8 . 3 9 9 . 7 0 0}$ & $\mathbf{1 0 0}$ & $\mathbf{1 . 7 7 4 . 8 0 0}$ & $\mathbf{1 0 0}$ \\
\hline
\end{tabular}

Fuente: Elaboración propia a partir de la operación estadística "El empleo de las personas con discapacidad" (INE).

\section{LA SITUACIÓN SOCIOLABORAL DE LAS PERSONAS CON DISCAPACIDAD}

A continuación, se analiza la situación laboral de las personas con discapacidad a partir de la información que contienen las encuestas de empleo de las personas con discapacidad elaboradas por el Instituto Nacional de Estadística (INE) en el período comprendido entre 2008 y $2015^{1}$.

\section{Empleo de las personas con discapacidad}

La evolución de la tasa de actividad de las personas con discapacidad desde 2008 (Gráfico 1) ha seguido un ritmo ligeramente ascendente hasta 2013 , año que marca un punto de inflexión en esta tendencia. En 2014 se produce un descenso de 3,8 puntos porcentuales, un descenso que no se observa en la tasa de actividad de las personas sin discapacidad, cuya tasa de actividad ha mantenido su crecimiento a pesar de la crisis iniciada en 2007.

${ }^{1}$ En la serie temporal de las estadísticas de "El empleo de las personas con discapacidad" existen dos rupturas metodológicas: 2008, 2009-2014 y 2015. No obstante, los datos del año 2014 han sido recalculados, según INE, utilizando la metodología 2015. 


\section{Gráfico 1.}

Evolución de las tasas de actividad en función de la discapacidad (2008-2015). (\%).

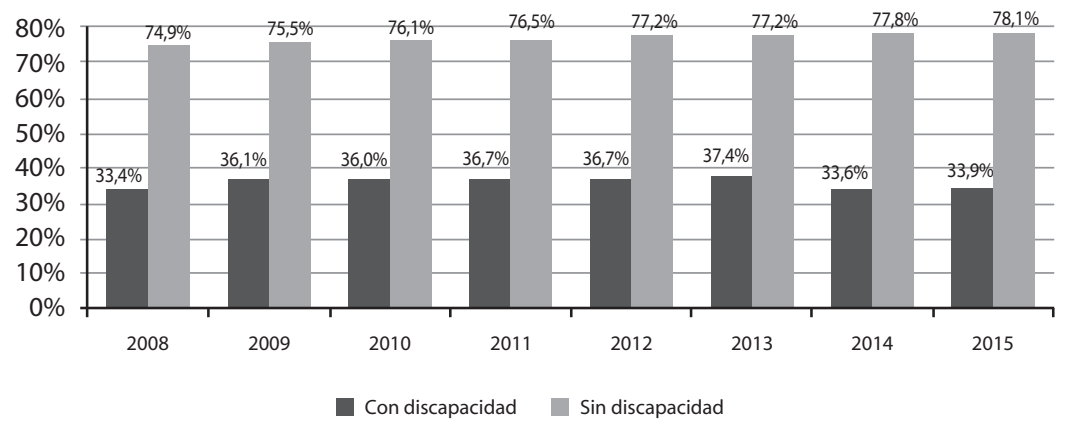

Fuente: Elaboración propia a partir de INE. El Empleo de las Personas con Discapacidad.

En cuanto a la ocupación del colectivo, la tasa de empleo de las personas con discapacidad en España en 2015 es del 23,4\% (Gráfico 2). Ello supone aproximadamente un tercio de la tasa de ocupación de las personas sin discapacidad. Al igual que entre estas últimas, la evolución del empleo ha sido negativa desde 2008, coincidiendo con el largo período de crisis económica.

\section{Gráfico 2.}

Evolución de las tasas de empleo en función de la discapacidad (2008-2015). (\%).

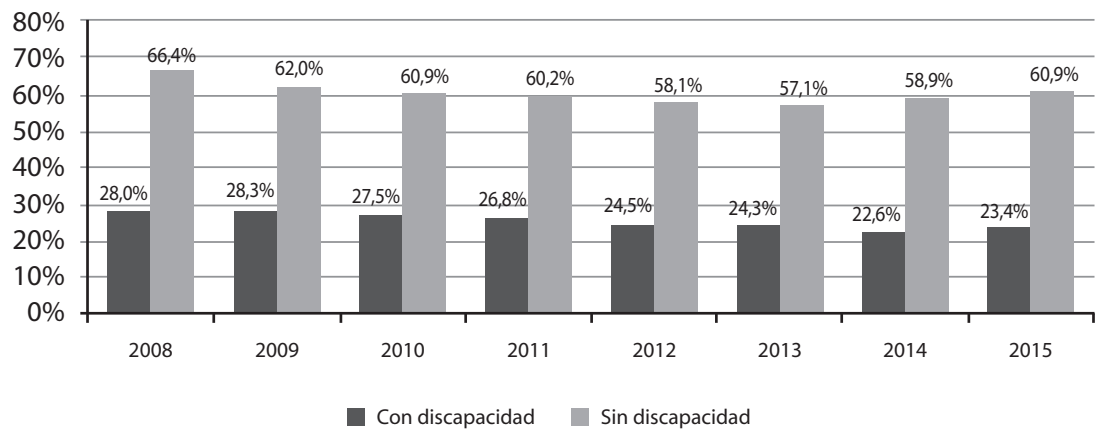

Fuente: Elaboración propia a partir de INE. El Empleo de las Personas con Discapacidad. 
En cuanto a la situación profesional es -mayoritariamentede asalariados. Sobre el total de las personas con discapacidad ocupadas, el empleo por cuenta propia apenas supone algo más del $11 \%$ en todo el período considerado, tal como se observa en el gráfico 3.

\section{Gráfico 3.}

Población con discapacidad ocupada por situación profesional (2008-2015). (\%).

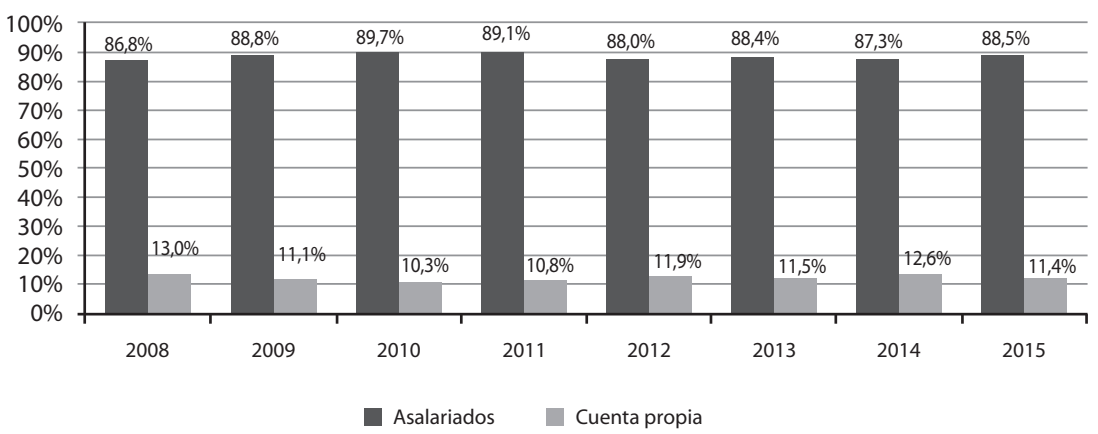

Fuente: Elaboración propia a partir de INE. El Empleo de las Personas con Discapacidad.

En relación al tipo de actividad desempeñada, está muy orientada al sector servicios. El $80,7 \%$ de las personas con discapacidad trabaja en actividades vinculadas con este sector en 2015. La ocupación en la industria es algo superior a un 12\% en ese mismo año y ha perdido efectivos desde 2008 (Gráfico 4). Por su parte, construcción y agricultura (con una ocupación del 3,8\% y $2,8 \%$ respectivamente en 2015 ) son sectores de carácter residual para este colectivo. 


\section{Gráfico 4. \\ Población ocupada con discapacidad por sector de actividad (2008-2015). (\%).}

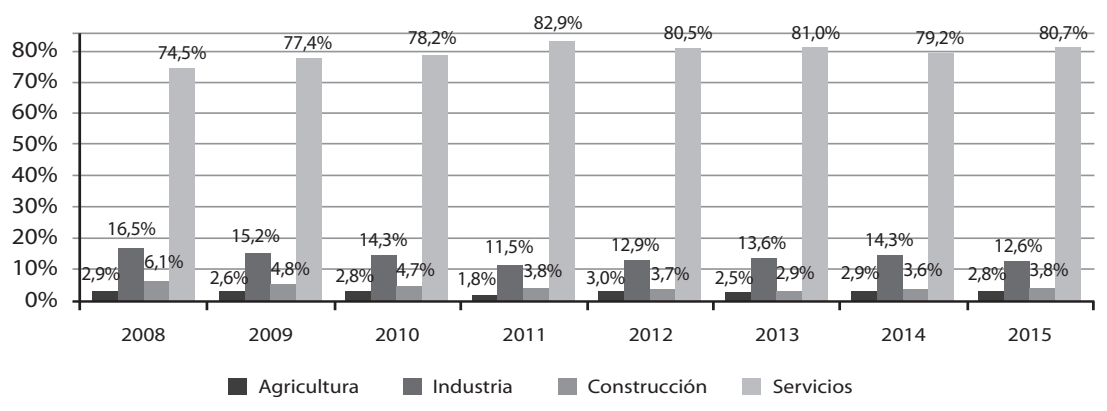

Fuente: Elaboración propia a partir de INE. El Empleo de las Personas con Discapacidad.

Más del $40 \%$ de estas personas desarrolla actividades que requieren baja cualificación y/o responsabilidad (Tabla 3). Actividades tales como la restauración, servicios personales, protección y venta ocupan en 2015 al $22,2 \%$ de las personas con discapacidad. Junto a éstas, las ocupaciones elementales $(20,7 \%)$ son las actividades que centran el empleo del colectivo. 
Tabla 3.

Población ocupada con discapacidad por ocupación CNO-11 (2011-2015). (\%).

\begin{tabular}{|l|c|c|c|c|c|}
\hline & $\mathbf{2 0 1 1}$ & $\mathbf{2 0 1 2}$ & $\mathbf{2 0 1 3}$ & $\mathbf{2 0 1 4}$ & $\mathbf{2 0 1 5}$ \\
\hline Directores y gerentes & 3,1 & 2,7 & 2,3 & 2,2 & 2,3 \\
\hline Técnicos y profesionales científicos e intelectuales & 12,4 & 13,2 & 12,9 & 11,0 & 12,0 \\
\hline Técnicos, profesionales de apoyo & 8,6 & 8,2 & 7,3 & 7,1 & 7,0 \\
\hline $\begin{array}{l}\text { Empleados contables, administrativos y otros em- } \\
\text { pleados de oficina }\end{array}$ & 17,5 & 15,6 & 17,1 & 18,3 & 17,0 \\
\hline $\begin{array}{l}\text { Trabajadores de los servicios de restauración, perso- } \\
\text { nales, protección y vendedores }\end{array}$ & 19,3 & 19,8 & 20,7 & 22,2 & 22,2 \\
\hline $\begin{array}{l}\text { Trabajadores cualificados en el sector agrícola, gana- } \\
\text { dero, forestal y pesquero }\end{array}$ & 1,5 & 3,1 & 3,1 & 2,9 & 2,6 \\
\hline $\begin{array}{l}\text { Artesanos y trabajadores cualificados de las indus- } \\
\text { trias manufactureras y la construcción (excepto } \\
\text { operadores de instalaciones y maquinaria) }\end{array}$ & 8,2 & 9,0 & 10,0 & 8,7 & 8,7 \\
\hline $\begin{array}{l}\text { Operadores de instalaciones y maquinaria, } \\
\text { y montadores }\end{array}$ & 8,2 & 8,9 & 7,9 & 7,8 & 7,6 \\
\hline Ocupaciones elementales & 21,2 & 19,5 & 18,8 & 19,8 & 20,7 \\
\hline Total & 100 & 100 & 100 & 100 & 100 \\
\hline
\end{tabular}

Fuente: Elaboración propia a partir de INE. El Empleo de las Personas con Discapacidad.

En resumen, respecto a la actividad laboral se observa la menor tasa de ocupación del colectivo de personas con discapacidad respecto a las que no la tienen (supone aproximadamente un tercio). Atendiendo a la situación profesional, las personas con discapacidad son mayoritariamente asalariadas y solo el $11 \%$ trabaja por cuenta propia y, por sectores, los servicios acaparan el mayor porcentaje del empleo en este colectivo, especialmente en tareas de media y baja cualificación.

\section{Desempleo y condiciones contractuales.}

La crisis ha pasado una costosa factura al colectivo de personas con discapacidad. Su nivel de paro se ha duplicado en el período de crisis económica, pasando de un 16,3\% en 2008 al $31,0 \%$ en 2015. Según los datos del INE, para este último año, esta tasa supera diez puntos porcentuales la de las personas sin discapacidad en España (Gráfico 5). 


\section{Gráfico 5. \\ Evolución de las tasas de paro \\ en función de la discapacidad (2008-2015). (\%).}

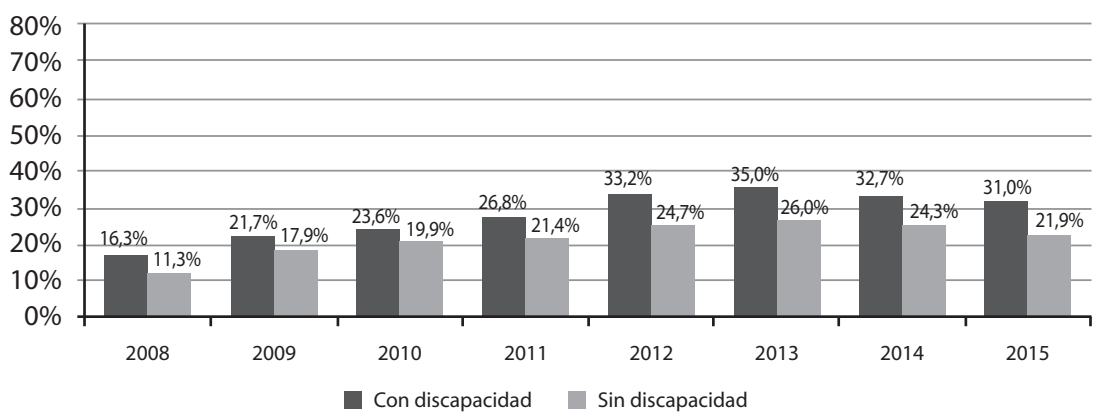

Fuente: Elaboración propia a partir de INE. El Empleo de las Personas con Discapacidad.

La contratación temporal es una forma muy frecuente de contratación en este colectivo (Gráfico 6), tanto en hombres como en mujeres. Entre los primeros, supera el $90 \%$ de los contratos celebrados en los últimos años, con una ligera ventaja en el caso de las mujeres. Como en el colectivo de personas sin discapacidad, resulta una fórmula de contratación cada vez más extendida, especialmente en un momento de crisis. No obstante, ha seguido una progresión ascendente en 2014 y 2015, una vez que la situación económica comenzó a mejorar.

\section{Gráfico 6.}

Contratos temporales por sexo (2008-2015). (\%).

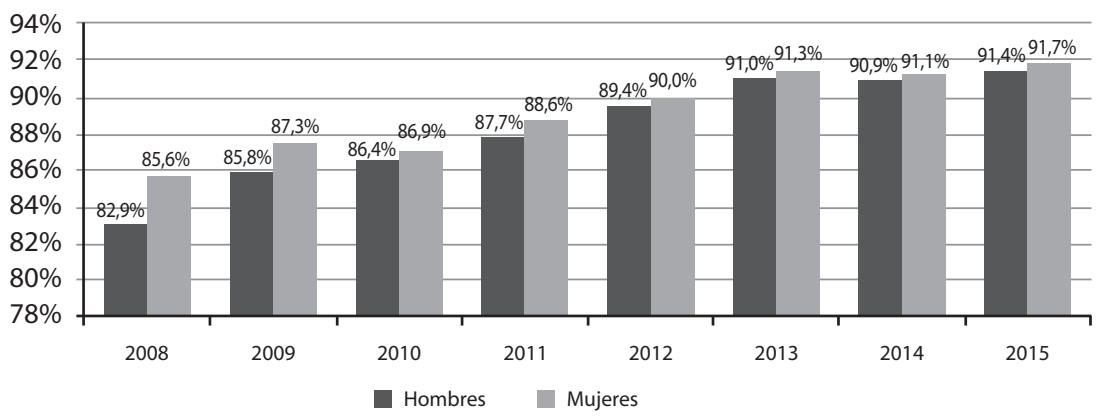

Fuente: Elaboración propia a partir de SEPE. Informe Anual del Mercado de Trabajo de las Personas con Discapacidad. 
También ha ido en aumento la contratación a tiempo parcial en paralelo al descenso en la contratación a jornada completa, que pierde más de diez puntos porcentuales entre 2008 y 2015 , evidenciando la precarización en las condiciones laborales de estas personas (Gráfico 7).

Gráfico 7.

Contratos por tipo de jornada (2008-2015). (\%).

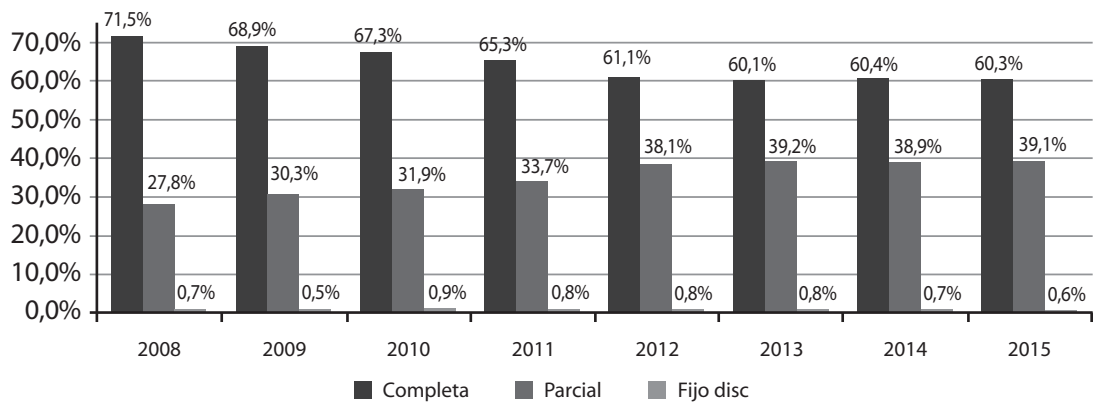

Fuente: Elaboración propia a partir de SEPE. Informe Anual del Mercado de Trabajo de las Personas con Discapacidad.

Fruto del ascenso en estas formas contractuales, el índice de rotación de este colectivo ha experimentado un aumento constante, lo que indica que el número medio de contratos por persona es mayor. En 2013 este índice supera los dos puntos y continúa en ascenso hasta el 2,1 en 2015, tal y como se observa en el Gráfico 8.

\section{Gráfico 8.}

\section{Índice de rotación de la contratación por persona (2008-2015).}

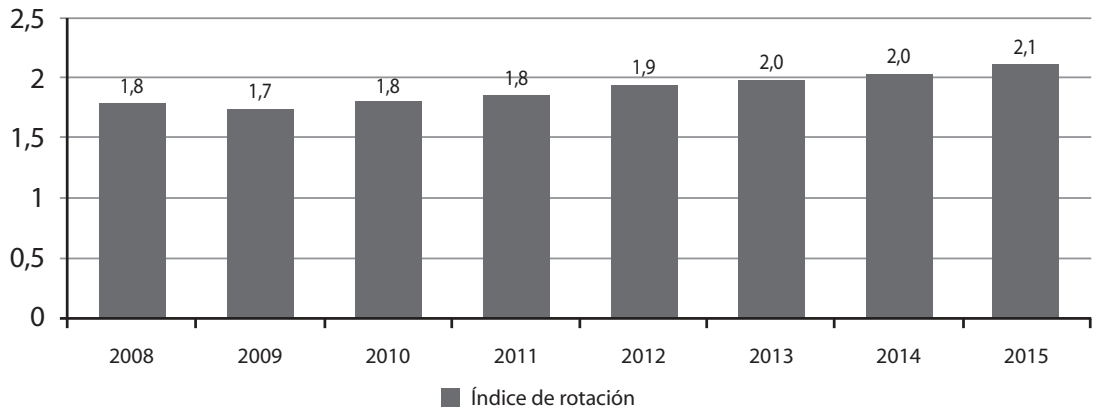

Fuente: Elaboración propia a partir de SEPE. Informe Anual del Mercado de Trabajo de las Personas con Discapacidad. 
Entre los efectos de la crisis, se cuenta la pérdida de capacidad adquisitiva de la población debido a la "congelación" y/o descenso salarial. En la tabla 4 se observa que la ratio salarial entre las personas con y sin discapacidad ha ido en descenso entre 2010 y 2014 (90,0 a 82,9), lo que implica que los salarios de uno y otro colectivo se han ido alejando entre sí. Dicha caída ha sido mayor entre las mujeres que entre los hombres, aunque la ratio de las primeras indica una mayor proximidad salarial entre uno y otro colectivo.

\section{Tabla 4.}

Ratio salarial entre personas con y sin discapacidad por sexo (2010-2014).

\begin{tabular}{|c|c|c|c|c|c|}
\hline Sexo & $\mathbf{2 0 1 0}$ & $\mathbf{2 0 1 1}$ & $\mathbf{2 0 1 2}$ & $\mathbf{2 0 1 3}$ & $\mathbf{2 0 1 4}$ \\
\hline Ambos sexos & 90,0 & 88,5 & 85,5 & 83,9 & 82,9 \\
\hline Hombres & 83,2 & 83,3 & 79,5 & 79,5 & 79,2 \\
\hline Mujeres & 98,0 & 93,7 & 91,5 & 87,9 & 84,5 \\
\hline
\end{tabular}

Fuente: Elaboración propia a partir de INE. El Salario de las Personas con Discapacidad.

\section{BARRERAS AL EMPRENDIMIENTO EN PERSONAS CON DISCAPACIDAD Y ACCIÓN DE LOS PODERES PÚBLICOS.}

Entre las barreras para la inclusión sociolaboral de personas con discapacidad caben citar el menor nivel educativo y una cualificación profesional más alejada de las demandas del sistema productivo; la existencia de prejuicios sociales relativos a su potencial laboral; las dificultades de accesibilidad; el incumplimiento de las medidas de discriminación positiva o el propio desarrollo de políticas pasivas que persuaden para el desarrollo de una alternativa laboral, según Mercado, Aizpurúa y García (2013). A estas barreras, los autores añaden dos factores como son, la dispersión de los recursos de información dirigidos al colectivo y, en segundo término, las expectativas personales y familiares con respecto a su participación en el mercado de trabajo.

Se han realizado algunos trabajos que han tratado, de manera más específica, las barreras al emprendimiento en el colectivo de 
personas con discapacidad. En este sentido, Moreno y Tejada (2015) coinciden en algunas de estas barreras en lo que se refiere al fomento del emprendimiento en el colectivo, a saber:

- Falta de financiación para la idea de negocio.

- Falta de formación, no tanto sobre aspectos relativos al emprendimiento como a la capacidad de autogestión y planificación ocupacional del emprendedor con discapacidad.

- Escasa implicación de los poderes públicos en el fomento del emprendimiento como alternativa profesional para el colectivo.

Por tanto, abogan por una legislación y desarrollo de medidas de apoyo activo al autoempleo y al emprendimiento de las personas con discapacidad, no tanto como un espacio exclusivo como por uno de participación en igualdad de condiciones y con un enfoque inclusivo de las estructuras creadas para el fomento de la actividad emprendedora.

Existen otras barreras de carácter específico que actúan sobre el desarrollo de la actividad emprendedora en personas con discapacidad. La propia Comisión Europea y la OCDE (2014), señalan:

- Falta de confianza y la configuración de aspiraciones más limitadas por parte de las personas con discapacidad.

- Condicionamientos en la percepción de los beneficios posibles, atribuyendo un mayor "riesgo" o inseguridad a la actividad por cuenta propia.

- Carencias formativas y competenciales vinculadas a la gestión empresarial, fruto de niveles educativos inferiores o de una carencia de experiencia profesional.

- Dificultades financieras y de recursos iniciales derivadas de la desventaja social en el nivel educativo y el acceso al mercado de trabajo.

- Posible discriminación por parte de los consumidores.

- Posible aumento de costes laborales, si requieren de más personal de apoyo para desarrollar la actividad.

- Ausencia de orientadores o asesores en materia emprendedora que puedan informar a las personas con discapacidad, fruto de una visión estereotipada de las mismas. 
Dada la existencia de estas barreras, cabe considerar la atención prestada por los poderes públicos. En materia de integración sociolaboral, del conjunto de personas con discapacidad, la acción de éstos acumula, en nuestro país, tradición democrática ya que el art.49 de la Constitución Española fija la responsabilidad de los poderes públicos de realizar una política de integración del colectivo. Así mismo, ya en 1982 se aprobó la Ley de Integración Social del Minusválido (LISMI) que configuró al colectivo de personas con discapacidad como objetivo de las políticas de empleo.

En la actualidad, la norma de referencia es el Real Decreto Legislativo 1/2013, de 29 de noviembre, por el que se aprueba el Texto Refundido de la Ley General de derechos de las personas con discapacidad y de su inclusión social. El art.37 de dicho texto, relativo a los tipos de empleo de las personas con discapacidad, establece que será finalidad de la política de empleo aumentar las tasas de actividad y de ocupación e inserción laboral de las personas con discapacidad, así como mejorar la calidad del empleo y dignificar sus condiciones de trabajo, combatiendo activamente su discriminación. Entre los tipos de empleo que el texto reconoce se encuentran el empleo ordinario, incluido los servicios de empleo con apoyo; el empleo protegido en centros especiales de empleo y en enclaves laborales, y el empleo autónomo. En este último sentido, el texto recoge que los poderes públicos en el ámbito de sus respectivas competencias, adoptarán políticas de fomento del trabajo autónomo de personas con discapacidad dirigidas al establecimiento y desarrollo de iniciativas económicas y profesionales por cuenta propia, o a través de entidades de la economía social. Sin embargo, cabe destacar la ausencia de concreción de actuaciones referidas al empleo autónomo, frente a las modalidades anteriores que se encuentran con un nivel de definición y concreción mucho más elevado.

Ballester, Cordero y Tomás (2014) destacan que el empleo autónomo se incluye, a efectos sistemáticos, como ámbito de integración de empleo ordinario. En este sentido, consideran que en esta doble vía se ha producido, tradicionalmente, una prevalencia en la integración de las personas con discapacidad en empresas ordinarias, y, de manera subsidiaria, se prevé la integración en el mercado de trabajo protegido a través de los centros especiales de empleo. Sin embargo, con el RD Legislativo 1/2013 la 
preferencia por el empleo ordinario se diluye y parece situarse al mismo nivel que el empleo protegido.

En relación a otros textos emanados de los poderes públicos, el Estatuto del Trabajo Autónomo recoge que la elaboración de la política de fomento del trabajo autónomo prestará especial atención a los colectivos de personas desfavorecidas, entre los cuales las personas con discapacidad ocupan un lugar preferente. Por su parte la Ley 56/2003 de Empleo establece que las Políticas Activas de Empleo tienen entre sus objetivos asegurar políticas adecuadas de integración laboral dirigidas a aquellos colectivos que presenten mayores dificultades de inserción laboral, como las personas con discapacidad. Y el Plan de Acción de la Estrategia Española sobre Discapacidad (2014-2020) tiene como uno de sus objetivos operativos en materia de empleo la promoción del emprendimiento de las personas con discapacidad.

El fomento del trabajo autónomo y del emprendimiento es una competencia asumida por las Comunidades Autónomas, generalmente en el marco de desarrollo de las políticas activas de empleo. Megino y Abril (2014), en un análisis de la regulación del trabajo autónomo y la discapacidad a nivel autonómico, concluyen sobre la falta de una regulación propia y específica para el colectivo de personas con discapacidad, ya que la mayor parte de la regulación autonómica se limita a determinadas referencias al mismo que no representan un tratamiento específico.

En definitiva, las personas con discapacidad, como colectivo de especial atención, están integradas como beneficiarias en la mayor parte de los textos que regulan las medidas del fomento del autoempleo y/o emprendimiento. No obstante, hay una carencia notable en el desarrollo de programas específicos. Además, existe cierta dispersión normativa en materia del fomento del autoempleo y/o emprendimiento de personas con discapacidad.

\section{DÉBIL MODELO DE INTEGRACIÓN SOCIOLABORAL A TRAVÉS DEL EMPRENDIMIENTO. RECOMENDACIONES PARA SU FORTALECIMIENTO.}

Las personas con discapacidad se constituyen como uno de los colectivos más vulnerables del mercado de trabajo, habiendo experimentado un ascenso del desempleo y unas condiciones 
contractuales más desfavorables, como son la contratación temporal y a tiempo parcial y la brecha salarial con respecto a las personas sin discapacidad, así como la mayor dificultad para incorporarse con éxito -y condiciones aceptables- al mercado de trabajo. Además, esta situación parece adquirir un carácter estructural o crónico, que hace que apenas se produzcan cambios a lo largo del tiempo.

Ante esta situación sociolaboral cabe preguntarse, en primer lugar, cómo se ha intervenido desde los poderes públicos y, en segundo lugar, si esta intervención ha contemplado el emprendimiento como una alternativa de desarrollo personal y profesional para las personas con discapacidad que favorezca su inclusión sociolaboral.

A la primera cuestión puede responderse de forma afirmativa, ya que existe una protección legal de los derechos de las personas con discapacidad y de su inclusión social. Además, el colectivo de personas con discapacidad es considerado de especial interés como destinatario de otras políticas, como por ejemplo las políticas de empleo en general.

No obstante, el sentido de la respuesta cambia en la segunda cuestión planteada. Las políticas de fomento del autoempleo o emprendimiento, tienen una menor tradición en nuestro país y ello ha provocado cierta amalgama de medidas en las que, si bien se contempla a las personas con discapacidad o el emprendimiento, adolece de un enfoque integrador de ambas vertientes, como sí se ha producido en el desarrollo de las medidas de integración laboral por cuenta ajena, bien sea a través de empleo ordinario o protegido. Existe pues, una definición y concreción institucional y social del modelo de integración sociolaboral para las personas con discapacidad por cuenta ajena, pero no se ha definido, ni desde las políticas de empleo ni desde las políticas de atención al colectivo, un modelo de integración sociolaboral a través del emprendimiento.

Ligado a los instrumentos políticos y su debilidad, cabría añadir un elemento previo y necesario para que se pudieran desarrollar verdaderas políticas de fomento del emprendimiento en las personas con discapacidad y es un análisis previo de las barreras de especial incidencia que el colectivo tiene para desarrollar una actividad emprendedora. En la superación de las mismas se centran algunas recomendaciones de actuación que favorezcan 
una mayor motivación entre las personas con discapacidad para emprender ya que, en última instancia, la empresa se crea "porque hay personas que tienen motivaciones para ello" (Campos, y Sanchís, 2014: 425).

La primera recomendación consiste en la mejora del nivel educativo en conjunto y solución de determinadas carencias competenciales de las personas con discapacidad.

La segunda se basa en favorecer la continuidad de este colectivo en el mercado de trabajo de forma que le permita acumular experiencia profesional.

La tercera recomendación se centra en mejorar el apoyo financiero a las personas con discapacidad junto con la mejora de la formación de los técnicos y los orientadores de empleo.

Una cuarta busca hacer compatible la actividad emprendedora con la protección y seguridad dadas por las políticas pasivas de empleo.

Finalmente, la última recomendación pretende potenciar un proceso de normalización de la discapacidad, de forma que se puedan superar las barreras impuestas por el propio entorno familiar y social a las personas con discapacidad.

Por tanto, en la necesidad de generar una visión integral sobre la discapacidad y el empleo, con una especial atención al que se presenta en forma de emprendimiento, así como en la obligación de superar las dificultades específicas de las personas con discapacidad para que el emprendimiento se entienda como una alternativa de desarrollo personal y profesional, se observan varias recomendaciones que se pueden agrupar en cuatro elementos clave como son la ayuda de la administración, la normalización de la discapacidad, el apoyo a la familia y la mejora del nivel educativo y las competencias asociadas con el emprendimiento.

\section{REFERENCIAS BIBLIOGRÁFICAS.}

Ballester Pastor, M.A.; Cordero Gordillo, V. y Tomás Jiménez, A. (2014). "Políticas de empleo (III): Políticas destinadas a colectivos especiales (I)" en Cardona Rubert, M.B. y Cabeza Pereiro, J. (Coords.): Políticas Sociolaborales (pp.327-374). Navarra: Aranzadi. 
Campos Climent, V. y Sanchís Palacio, J.R. (2014): "Políticas de fomento del autoempleo" en en Cardona Rubert, M.B. y Cabeza Pereiro, J. (Coords.): Políticas Sociolaborales (pp.423-462). Navarra: Aranzadi.

Megino Fernández, D. y Abril Larraínzar, M.P. (2014). "Previsiones legales sobre trabajo autónomo y discapacidad a nivel autonómico" en Barrios Baudor, G.L. (Coord.): Trabajo autónomo y discapacidad (pp. 89-128). Madrid: Ramón Areces.

Mercado García, E.; Aizpurúa González, E. y García Vicente, L. M. (2013): "Avanzando hacia la igualdad de oportunidades en la inclusión socio-laboral de las personas con discapacidad", en Cuadernos de Trabajo Social, vol. 26, núm. 1, pp. 95-104.

Moreno Rodríguez, R. y Tejada Cruz, A. (2015). "El emprendimiento como alternativa laboral de las personas con discapacidad: importancia de la ocupación productiva sobre la calidad de vida", en I Congreso Nacional de Empleo y Discapacidad (Libro de Actas), Granada, 5-6 de marzo, pp. 50-57.

OECD/European Union (2014). Policy Brief on Entrepreneurship for People with Disabilities. Luxemburgo: Publications Office of the European Union.

OMS. Organización Mundial de la Salud (1997). "Clasificación Internacional de Deficiencias, Discapacidades y Minusvalías", IMSERSO, Madrid.

ONCE, Fundación. Organización Nacional de Ciegos Españoles (2013). El sector de la discapacidad: realidad, necesidades y retos futuros Análisis de la situación de la población con discapacidad y de las entidades del movimiento asociativo y aproximación a sus retos y necesidades en el horizonte de 2020. Colección Cermi.es: Madrid. 